\title{
Programming the group behaviors of bacterial communities with synthetic cellular communication
}

\author{
Wentao Kong ${ }^{1,2 \dagger}$, Venhar Celik ${ }^{1,2 \dagger}$, Chen Liao ${ }^{1,2}$, Qiang Hua ${ }^{3}$ and Ting Lu 1,2, $^{*}$
}

\begin{abstract}
Synthetic biology is a newly emerged research discipline that focuses on the engineering of novel cellular behaviors and functionalities through the creation of artificial gene circuits. One important class of synthetic circuits currently under active development concerns the programming of bacterial cellular communication and collective population-scale behaviors. Because of the ubiquity of cell-cell interactions within bacterial communities, having an ability of engineering these circuits is vital to programming robust cellular behaviors. Here, we highlight recent advances in communication-based synthetic gene circuits by first discussing natural communication systems and then surveying various functional engineered circuits, including those for population density control, temporal synchronization, spatial organization, and ecosystem formation. We conclude by summarizing recent advances, outlining existing challenges, and discussing potential applications and future opportunities.
\end{abstract}

Keywords: Synthetic biology; Gene circuits; Bacterial communities; Cellular communication; Collective behaviors; Dynamics

\section{Background}

Synthetic biology is a newly emerged research discipline that focuses on the engineering of novel cellular behaviors and functionalities. Since the launch of the field in 2000 $[1,2]$, a wide range of synthetic gene devices have been created, including switches [3-9], oscillators [10-13], memory elements $[7,14,15]$, and communication modules $[13,16-18]$, as well as other electronics-inspired genetic devices, such as digital logic gates [19-22], pulse generators [23], and filters [24,25]. With designed cellular behaviors and functionalities, engineered circuits have been exploited to understand biological questions and to address various real-world problems [26]. The field has shown tremendous potential for biomedical, environmental, and energy-related applications [27]. For example, towards biomedical applications, engineered genetic circuits contribute to the understanding of disease mechanisms,

\footnotetext{
* Correspondence: luting@illinois.edu

${ }^{\dagger}$ Equal contributors

'Department of Bioengineering, University of Illinois at Urbana-Champaign, 1304 W Springfield Avenue, Urbana, IL 61801, USA

${ }^{2}$ Institute for Genomic Biology, University of Illinois at Urbana-Champaign, 1206 W Gregory Drive, Urbana, IL 61801, USA

Full list of author information is available at the end of the article
}

provide novel diagnostic tools, enable economic production of therapeutics, and enable the design of novel treatment strategies for various diseases including cancer, metabolic disorders, and infectious diseases [28,29].

In the last few years, the advances of synthetic circuits have been further expedited, empowered by recent breakthroughs in genetic engineering techniques such as novel DNA assembly [30-33] and genome editing tools [34-37], advances in methodologies including those for rational circuit design and optimization [38-40], and quick enrichment of parts and elements $[41,42]$. As a result, synthetic biologists are now in a position to engineer desired cellular phenotypes in a larger, faster, and cheaper fashion.

One important class of synthetic circuits that are under active development concerns the programming of bacterial cell-cell communication and the group behaviors of communities [43-48]. Successful examples include gene constructs responsible for cellular density control [18], spatiotemporal patterning $[13,16,49,50]$, and ecosystem formation [51,52]. The engineering of community-based circuits is essential and invaluable towards the implementation of complex but robust cellular functionality because of the following reasons: First, although microbes are 
single cell organisms, they are present dominantly in the form of communities in nature and in live bodies, such as biofilms [53,54] and the human microbiome [55,56]. Second, microbial physiology and functionality are strongly correlated with their forms - for instance, bacterial antibiotic resistance is distinct when cells are in planktonic forms and biofilm forms $[57,58]$. Third, recent advances in the biotechnological industry have clearly shown that microbial consortia may provide many compelling advantages in producing products of interest and controlling fermentation processes $[59,60]$.

We are thus motivated in this article to overview the advances of synthetic gene circuits towards the programming of bacterial cellular communication and community behaviors. We will first discuss basic communication modules that confer cell-cell coordination in communities. We will then overview various functional gene circuits that enable the implementation of desired dynamic group behaviors, including those for population density control, temporal synchronization, spatial organization, and ecosystem formation. We will conclude by summarizing recent advances and discussing existing challenges, potential applications, and future opportunities.

Although not discussed here, it is important to note that there has been considerable progress in developing synthetic cellular communication in eukaryotes such as mammalian cells and yeast, which has been surveyed in the literature $[51,61,62]$.

\section{Review}

\section{Basic communication modules}

Despite their species diversity, bacteria often utilize similar signaling systems for the implementation of their group behaviors [63,64]. For instance, quorum sensing (QS) is prevalent in bacteria for coordinating their group behaviors such as bioluminescence [65], biofilm formation [66], pathogenesis [67] and antibiotic synthesis [68-70].

\section{Bacterial communication via nonvolatile signaling molecules}

In Gram-negative bacteria, acyl-homoserine lactones (AHLs) are commonly used as QS molecules for intraspecies communication. These molecules are composed of a homoserine lactone ring with an acyl chain of $\mathrm{C} 4$ to C18 in length $[71,72]$. AHL molecules are synthesized by the LuxI family synthases and detected by the corresponding LuxR-type receptors [73]. One canonical example of this class of communication is the QS system discovered in the bioluminescent marine bacterium Vibrio fischeri [74]. As shown in Figure 1A, LuxI, the autoinducer synthase, produces the AHL molecule $3 \mathrm{OC}_{6} \mathrm{HSL}$ that can diffuse freely across the cell membrane and accumulate with the increase of cell density. Once the AHLs reach a threshold concentration, they form a complex with the LuxR receptor and activate the transcription of the downstream genes ( $l u x I$ and $l u x R$ in this case). A positive feedback regulatory architecture arises here from the self-activation of LuxI synthesis to facilitate the synchronization of the cellular population. Similar to $V$. fischeri, many other Gram-negative bacteria also possess QS systems, including the LasI/LasR and RhlI/RhlR systems in Pseudomonas aeruginosa [75], the CarI/CarR system in Erwinia carotovora [76], and the Esal/EsaR system in Pantoea stewartii [77]. From an engineering perspective, these QS systems can be decomposed into two separate modules with one for signal production and the other for signal detection and response - when engineered in different cells, the two functional modules will confer communications between the two cells as shown in Figure 1C.

In Gram-positive bacteria, modified oligopeptides often serve as the signaling molecules for cellular communication with the cooperation of two-component systems. One classic example of this type of system is the Agr system in Staphylococcus aureus (Figure 1B) [78]. Here, the auto-inducing peptide (AIP) precursor, encoded by the gene $a g r D$, is modified on its thiolactone ring and exported by AgrB protein. Upon the binding of AIP with the transmembrane protein AgrC, the transcriptional factor AgrA inside the cell is phosphorylated and then activated, which leads to the induction of the transcription of the downstream genes ( $a g r B / D / C / A$ here). In addition to the Agr system, there are many communication systems based on auto-inducing peptides, such as the fsr system in Enterococcus faecalis [79], the Com system of Streptococcus pneumonia [80], and the nisRK system in Lactococcus [70]. To program collective behaviors in Gram-positive bacteria, a modular partition of those AI systems can thus been exploited (Figure 1D).

Other than the QS and AIP systems that are primarily present in intra-species communication, there are interspecies communication systems that coordinate cellular behaviors over multiple bacterial species. One such example is the communication systems mediated by the universal signaling molecule autoinducer-2 (AI-2), a furanosyl borate diester synthesized by LuxS from $S$-adenosylmethionine and present in roughly half of all sequenced bacterial genome $[81,82]$. Towards programmable behaviors in multiple bacterial species, AI-2 is hence an ideal candidate for exploitation.

\section{Bacterial communication via volatile and gas molecules}

The adoption of nonvolatile molecules, such as AHLs and AIs, as the broadcast signal enables cellular coordination across various species. However, communications via those molecules require the presence of the both sender and receiver species in the same liquid environments or in gel-like setting within a short distance to allow for diffusion of signaling molecules. Volatile molecules, in contrast, can diffuse through air and circumvent the need of 
(A)

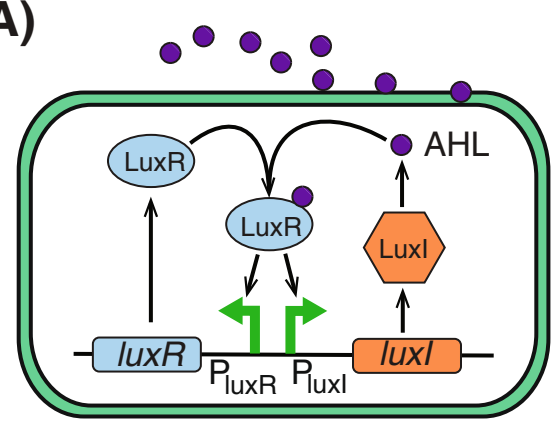

(B)

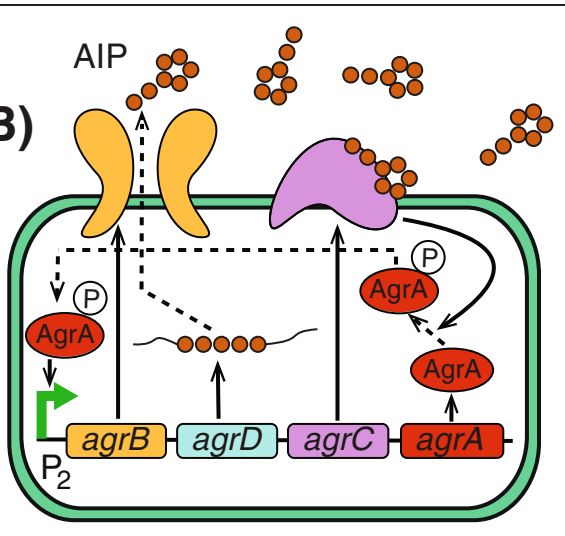

(C)
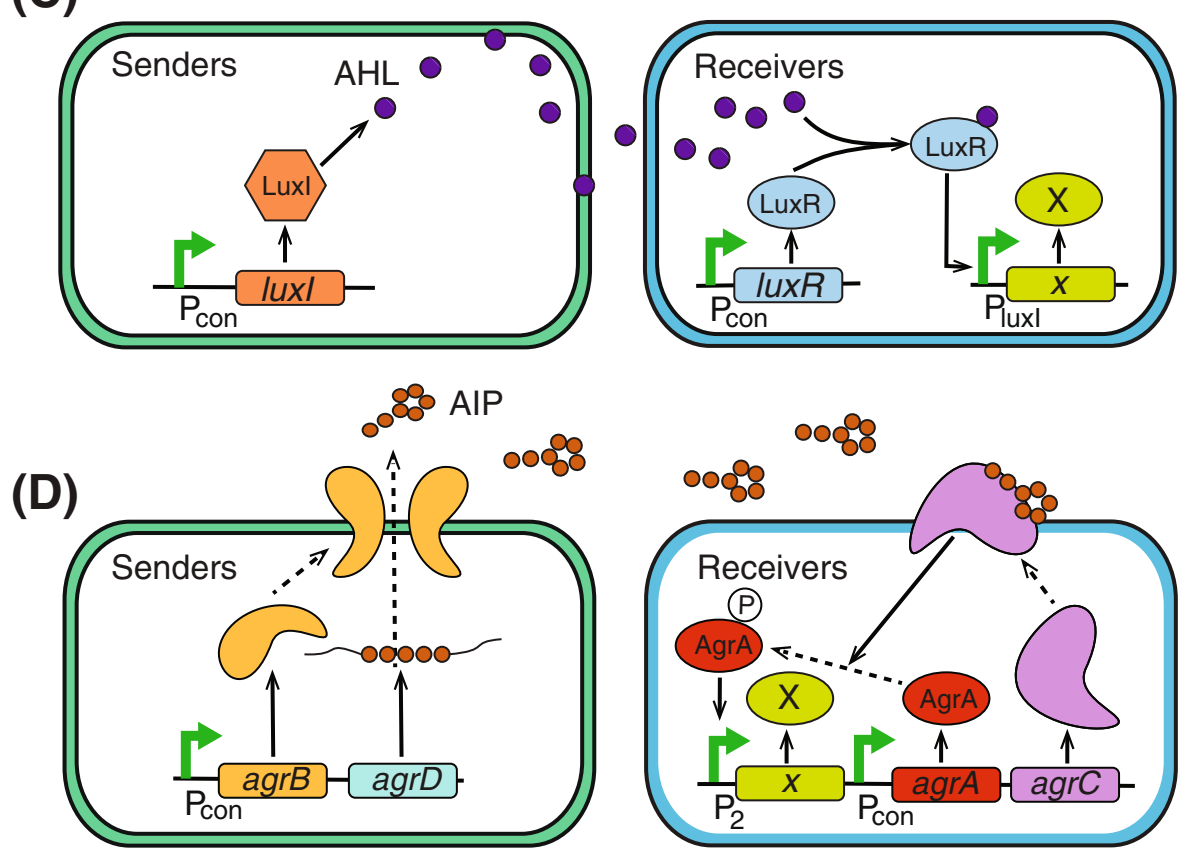

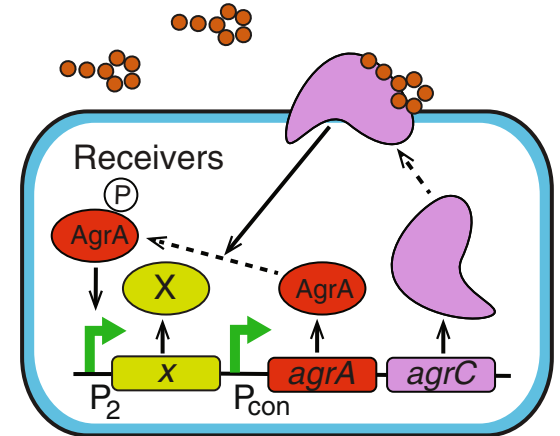

Figure 1 Cellular communication in bacteria. (A) The LuxI/LuxR quorum sensing (QS) system in the Gram-negative bacterium Vibrio fischeri. The system consists of the genes luxl and luxR and the cognate promoter $P_{\text {luxl }}$ and $P_{\text {luxR. }}$. Its signaling molecule is the acyl-homoserine lactone (AHL) 3OC 6 HSL. (B) The Agr QS system in the Gram-positive bacterium Staphylococcus aureus. It consists of the genes, agrD, agrB, agrC, and agrA and the cognate promoter $\mathrm{P}_{2}$. The auto-inducing peptide (AIP) is the signaling molecule of the system. (C) An engineered communication module adapted from the wild-type Luxl/LuxR system in (A). By expressing / I XXI, the sender cell (left) produces the signal AHL that diffuses to the extracellular milieu and further into the receiver cell (right) to alter the expression of the downstream genes $X$. (D) A synthetic communication module built from the Agr system in (B). The sender cell (left) produces and secretes the signaling molecule AIP that is sensed by the receiver cell (right), resulting in the expression shift of the gene $X$ in the receiver cell.

physical mediating settings for signaling, allowing for more versatile, rapid, and large-scale communications of communities.

Weber et al. recently established a communication system that utilizes acetaldehyde as signaling molecules [51]. In their study, a bacterial strain (sender) was engineered to constitutively express alcohol dehydrogenase $(\mathrm{ADH})$, an enzyme that converts ethanol in the medium to acetaldehyde. Due to its low boiling point $\left(21^{\circ} \mathrm{C}\right)$, acetaldehyde volatized and was broadcast to neighboring cells (receiver) via air to trigger the expression of genes controlled by the cognate acetaldehyde-inducible promoters. Therefore, the sender cells produced a concentric gradient of acetaldehyde that induced the dose-dependent gene expression of the receiver cells with the expression level defined by the distance between the sender and receiver cells.

In another example, Hasty and colleagues constructed $n d h$-2, a gene encoding NADH dehydrogenase II (membrane-bound respiratory enzyme), into an Escherichia coli 
strain to confer the production of hydrogen peroxide $\left(\mathrm{H}_{2} \mathrm{O}_{2}\right)$ [83]. $\mathrm{H}_{2} \mathrm{O}_{2}$ is a thermodynamically unstable chemical compound and is able to enter neighboring cells quickly to alter their redox state and inactivate ArcAB, resulting in the shift of the activity of the corresponding downstream genes. Through the exploitation of $\mathrm{H}_{2} \mathrm{O}_{2}$, a novel route of airborne signaling molecule was created for fast and large-scale colony coordination.

\section{Other communication mechanisms}

In addition to the common signaling mechanisms discussed above, bacteria also exploit a wide range of alternative approaches for communications, such as quinolone signal [84], diffusible signal factor [85], cyclic dipeptide [86], diketopiperazines [86,87], and others [88,89]. One such representative mode of signaling is the use of indole, an aromatic heterocyclic organic compound that is produced by over 85 species of Gram-positive and Gram-negative bacteria and used as an extracellular signal for global coordination of various bacterial species [90]. Although little of those mechanisms have been explored for synthetic biology applications, the broad spectrum of signaling systems provides a rich reservoir for engineering multicellular functionality.

\section{Dynamic group behaviors of bacterial communities via engineered communications}

Cellular communications enable the coordination of single cells by sending and sensing the states of individuals. Inspired by this natural capability of bacteria, synthetic biologists have developed a set of engineered bacterial populations with their group behaviors programmed from designed artificial cell-cell communications.

\section{Population density control}

The first communication-based synthetic circuit was built by You et al. in 2004 with the goal of creating a dynamic, autonomous regulation of the cell density of an E. coli population [18]. As illustrated in Figure 2A, the Lux system from $V$. fischeri was introduced to construct cell-cell communication and was coupled to cell survival and killing via the $\mathrm{CcdA} / \mathrm{B}$ toxin system. Here, the LuxI protein catalyzes the synthesis of a small, diffusible AHL signaling molecule, $3 \mathrm{OC}_{6} \mathrm{HSL}$, which accumulates in the extracellular milieu and the intracellular environment as the cell density increases. When cells reach a sufficient density, the AHL binds to LuxR and forms the LuxR/AHL complex that activates the expression of the killer protein LacZ $\alpha-C c d B$, leading to cell death. On the other hand, cell death can cause a reduction of total population density and hence the level of AHL production, which in turn allows the population to recover after killing. The continuous production and degradation of AHL make the cell density approach a steady

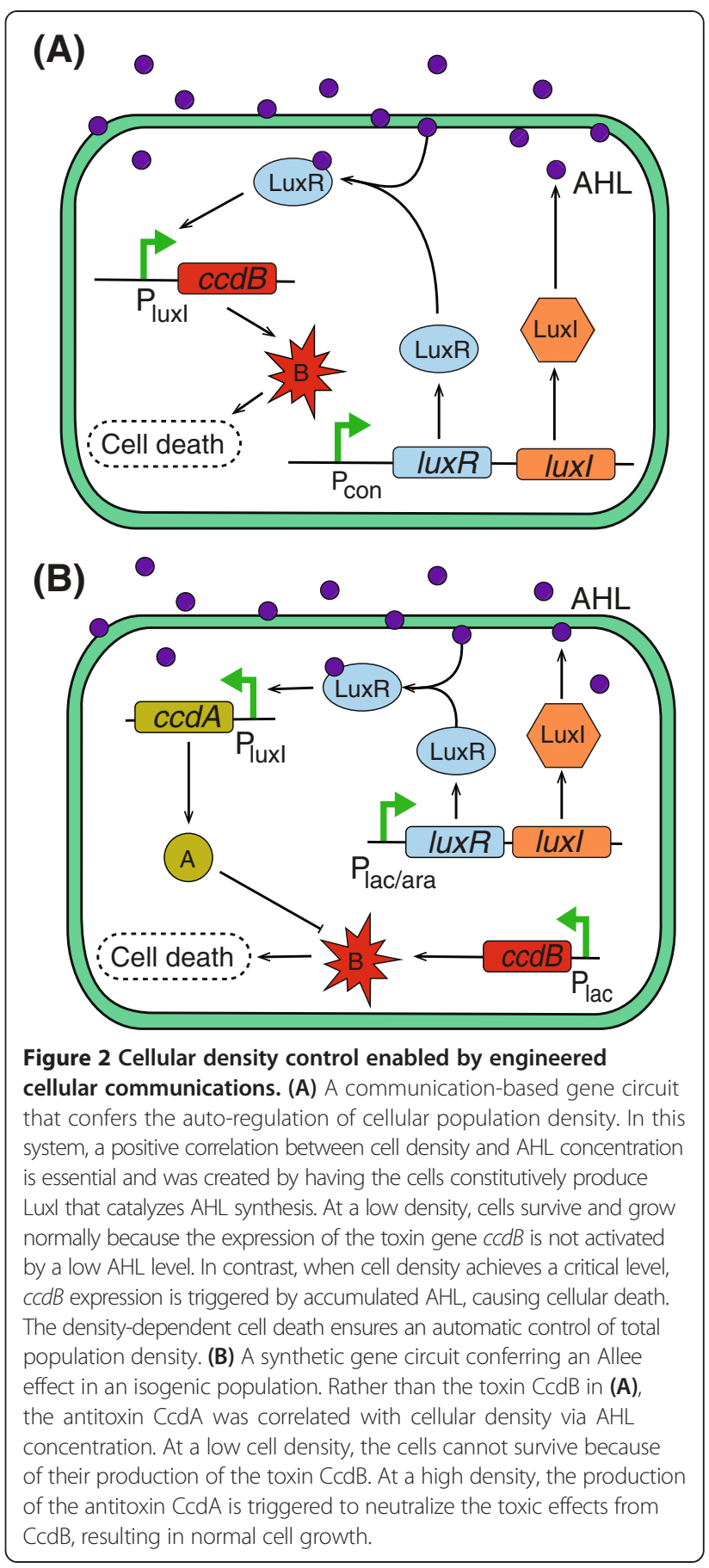

state. Indeed, a stable cell density was maintained for more than $30 \mathrm{~h}$ with the variation within less than $5 \%$ in the study. This density control circuit laid a foundation for using cellular communications to program bacterial communities, allowing the extension of the control of population dynamics to the engineering of more sophisticated synthetic ecosystems.

In a recent work, Smith et al. utilized the density control circuit constructed above to create an artificial Allee effect 
in E. coli populations [91]. The Allee effect is a biological phenomenon characterized by a correlation between population density and the mean individual fitness of a population [92]. To create such an effect, a synthetic gene circuit was constructed to contain the LuxI/LuxR system and the $\mathrm{CcdA} / \mathrm{B}$ toxin-antitoxin system (Figure $2 \mathrm{~B}$ ). In this setting, the expression of LuxR/LuxI and CcdB (killer) is under the control of $\mathrm{P}_{\text {lac/ara }}$ promoter, while CcdA (rescue) was regulated by the cell density-dependent $\mathrm{P}_{\text {lux }}$ promoter. When IPTG induction is on, the cellular population growth rate is negative if the initial cell density is less than the critical value $\left(C_{\text {crit }}\right)$ at which $\operatorname{CdA}$ expression is not activated. However, if the initial cell density is above $C_{\text {crit }}$, AHL activates the production of LuxR and further drives the production of $\mathrm{CcdA}$ which rescues the population by inhibiting the toxicity of CcdB. An Allee effect population was thus established to have a negative fitness below a threshold of cell density but a positive fitness when the density is beyond the threshold. This study provided new implications of engineered cellular communication for controlling invasive species and the spread of infectious diseases.

\section{Temporal synchronization}

Complex cellular behaviors, such as biofilm formation and host invasion, often require the temporal coordination and collective action of cellular populations [93,94]. Towards this need, engineered communications offer a powerful solution.

In a recent study, Hasty and colleagues reported the development of an artificial gene circuit that synchronizes the oscillation of gene expression in individual cells [13]. Figure 3A shows their circuit design based on the QS elements of $V$. fischeri (luxI, luxR) and Bacillus thurigensis (aiiA). The AHL $3 \mathrm{OC}_{6} \mathrm{HSL}$, synthesized by LuxI, binds to transcriptional factor LuxR to form a complex (LuxR-AHL) that activates the expression of luxI, which leads to a positive feedback loop in regulation. At the same time, the LuxR-AHL complex also activates the expression of aiiA, a gene encoding the AHL degradation enzyme, which leads to a negative feedback loop in regulation. The dual positive and negative feedback loops drive the sustained oscillation of gene expression of individual cells, and in the meantime, the signaling molecule AHL confers the synchronization of individual oscillations. Using a custom-tailored microfluidic device, the authors were, for the first time, able to establish and tune synchronized oscillations of an entire cellular population (thousands of cells). Compared with the single cell oscillators developed by the same group [11] and other researchers $[1,10]$, the engineered cellular communication indeed conferred the synchrony of cellular gene expression dynamics at a robust and yet tunable fashion.

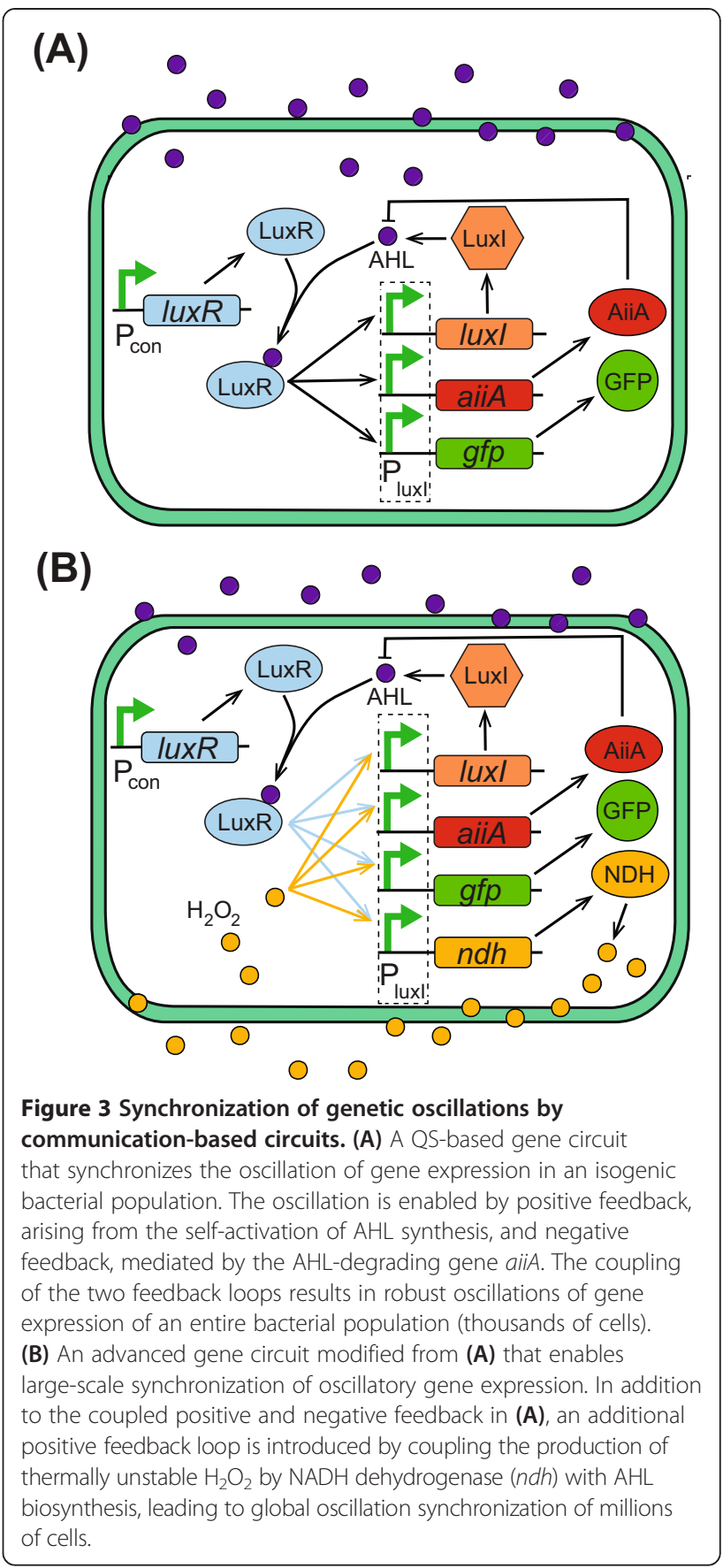

Building on their success of the synchronized oscillator, the same group further advanced to create a more sophisticated genetic network that is capable of synchronizing oscillatory gene expression of populations across multiple spatial scales [83]. As illustrated in Figure 3B, the researchers placed a copy of the $n d h-2$ gene, which encodes NADH dehydrogenase II, under the control of an additional copy of $\mathrm{P}_{\text {lux }}$ promoter (compared with Figure 3A). The NDH-2 produces a low level of $\mathrm{H}_{2} \mathrm{O}_{2}$ that vapors and passes through the walls of the oxygen-permeable 
polydimethylsiloxane (PDMS) chips. Driven by the oscillation of gene expression mediated by the AHL-based synchronized oscillation circuit, $\mathrm{H}_{2} \mathrm{O}_{2}$ was periodically produced and exchanged between the cells within individual chambers. When entering cells, $\mathrm{H}_{2} \mathrm{O}_{2}$ changes the redox state of the cells and inactivates their lux promoter binding protein $\mathrm{ArcAB}$, causing the global activation of the lux promoter of the cells in different chambers. As a result, thousands of oscillating colony 'biopixels' (approximately 2.5 million cells) were synchronized over centimeter-length scales through the use of synergistic intercellular coupling involving both quorum sensing within a colony and gas-phase redox signaling between colonies. As a proof-of-concept application, this system was further employed to sense arsenic in environments via differential modulations of the period of the oscillatory cells that resemble a liquid crystal display (LCD)-like macroscopic clock.

\section{Spatial organization}

One of the most fascinating aspects of biological systems is their ability to generate complex but highly reproducible organisms through differential spatial patterning of morphogens across isogenic cells [95]. Towards the ultimate goal of biological engineering for creating desired tissues, organs, or even entire organisms, one critical step is to develop an engineering strategy that enables robust spatiotemporal pattern formation of living cells. Engineered cellular communications hold a great promise towards this goal, in addition to their roles in conferring temporal coordination of cellular behaviors.

In fact, synthetic biologists have already made several interesting attempts through the exploitation of artificial communication-based gene circuits. For instance, Sohka et al. constructed a circuit implementing Wolpert's French flag model [96], enabling the determination of cell fates in a concentration-dependent manner [25]; Payne et al. created a circuit that allows self-organized pattern formation without morphogen gradients in bacteria [97]; Basu et al. engineered a band detector that allows for differential response of gene expression according to the local concentration of AHL, creating a bull's eye-like spatiotemporal pattern [16].

One elegant example for this line of applications is the programming of bacterial stripe patterns by Liu and Coworkers [49]. As shown in Figure 4A, the gene circuit consists of two functional parts: density-sensing module and motility-control module. The density-sensing module centers on the LuxI/LuxR QS system that enables the synthesis and excretion of the AHL and the activation of the

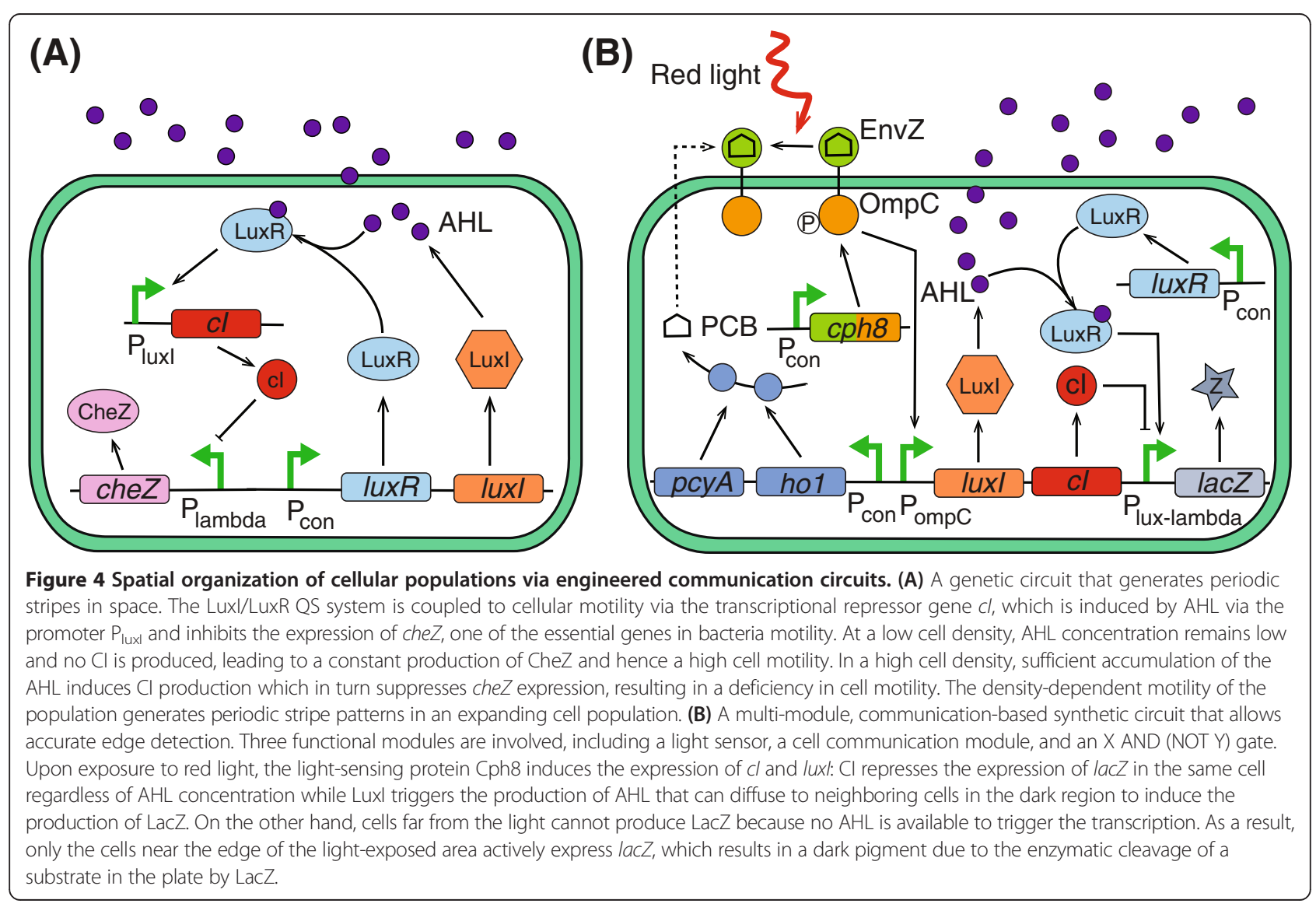


downstream gene $c I$ when cell density is sufficiently high. The motility-control module is based on the bacterial motility system that is regulated by the transcription of cheZ. Upon the replacement of the wild-type cheZ with an inducible version (che $Z$ is under the control of the cI-repressed $\mathrm{P}_{\text {lambda }}$ promoter), cellular motility becomes regulated by the expression of $\mathrm{cI}$. With the coupling of the two modules, engineered $E$. coli populations were able to form robust but tunable periodic stripes of high and low cell densities sequentially and autonomously. These results established cellular motility as a simple route to create recurrent spatial structures without the need for an extrinsic pacemaker. As a novel mechanism, it offered an alternative solution for the formation of biological spatial patterns that is distinct from the well-acknowledged Turing mechanism [98].

In addition to autonomous pattern formation, the QS-based communication mechanism can also be applied to detect complex spatial signals. Tabor et al. recently developed a multi-module gene circuit system for edge detection, a signal processing algorithm common in artificial intelligence and image recognition [99]. As illustrated in Figure $4 \mathrm{~B}$, the biological edge detection algorithm is composed of three modules: a dark sensor (NOT light), cell-cell communication cassette, and an X AND (NOT Y) genetic logic. The darker sensor was engineered based on the light-sensitive protein $\mathrm{Cph} 8$, a chimeric sensor kinase. With the covalent association of chromophore phycocyanobilin produced from heme via ho1 and pcyA $[100,101]$, Cph8 is able to activate the ompC promoter $\left(\mathrm{P}_{\text {ompC }}\right)$ by transferring a phosphoryl group to the response regulator OmpR. However, in the presence of red light, the kinase activity of Cph8 is inhibited, which precludes the transcription from $\mathrm{P}_{\text {ompC }}$ and causes a NOT light transcriptional logic gate. The cell-cell communication was implemented through the Lux QS system and was used to convert light information into spatial distribution of AHL. With the incorporation of the converter $\mathrm{CI}$ and the hybrid promoter $\mathrm{P}_{\text {lux-lambda }}$, the state of $\mathrm{P}_{\mathrm{ompC}}$ is converted via an $X$ AND (NOT Y) logical operation into the state of the promoter $\mathrm{P}_{\text {lux-lambda }}$ which is displayed via the production of LacZ that produces black pigment. Upon the loading of the programs, a lawn of isogenic E. coli populations was able to sense an image of light, communicate to identify the light-dark edges, and visually present the result of the computation.

\section{Ecosystem formation}

Artificial cellular communications can enable not only the coordination of isogenic cell populations but also heterogeneous ecosystems that are composed of multiple species. You and co-works recently developed two gene circuits into a predator-prey ecosystem that consists of two E. coli populations [52].
Figure $5 \mathrm{~A}$ shows the design of the ecosystem that involves two QS modules, LuxI/LuxR from $V$. fischeri and LasI/LasR from $P$. aeruginosa, for two-way communications. The predator cell (top) produces and secretes the AHL $3 \mathrm{OC}_{12} \mathrm{HSL}$ that induces the expression of the toxin gene $c c d B$ in the prey cell (bottom), leading to the death of the prey. In the meantime, the prey produces another AHL molecule, $3 \mathrm{OC}_{6} \mathrm{HSL}$, which rescues the predator by inducing the production of antitoxin CcdA that neutralizes the toxin from CcdB. With appropriate modulations of the system parameters, the researchers were able to create a bacterial version of predation with different population dynamics generated, including extinction, coexistence, and oscillation. Similar to this work, another bidirectional intercellular communication network was also engineered by Brenner et al. [50], in which the LasI/ LasR and RhlI/RhlR QS systems from $P$. aeruginosa were adopted to create a two-species microbial consensus consortium. In that ecosystem, the gene expression of any of the two species mutually depends on the presence of the other.

Beyond predation and consensus, designer cellular communications can be used to create a wide spectrum of inter-species interactions. As revealed by metagenomics and $16 \mathrm{~S}$ pyrosequencing, microbial interactions in nature such as biofilms and the microbiome are extremely complicated and diverse - for instance, there can be parasitism, predation, commensalism, mutualism, competition, and amensalism within a single pair of species [102]. As one of the earliest efforts towards the programming of complicated cellular consortia, Weber and Fussenegger developed a set of pairwise interactions between E. coli and Chinese hamster ovary $(\mathrm{CHO})$ cells [51].

As illustrated in Figure 5B, the designs of the ecosystems center on an airborne transmission of the transcription system that allows one species (E. coli) to convert ethanol into volatile acetaldehyde and broadcast this airborne signal (boiling point: $21^{\circ} \mathrm{C}$ ) to another species ( $\mathrm{CHO}-\mathrm{K} 1$ cell line) for the activation of functionally specific, rationally engineered genes. The commensal ecosystem (top) was created by constructing an $E$. coli strain capable of converting ethanol into acetaldehyde for air broadcast and placing a neomycin resistance gene (neo) under the control of an acetaldehyde-induced promoter $\left(\mathrm{P}_{\text {air }}\right)$ in a CHO-K1 cell line. In addition, secreted alkaline phosphatase (SEAP) was used as a reporter of the CHO-K1 cells. When cultivated proximate to synthetic $\mathrm{CHO}-\mathrm{K} 1$, the engineered E. coli cells confer survival of the mammalian cells while keeping their own growth unaffected by the mammalian cells cultured in a separate dish. The amensal ecosystem (middle) was synthesized by cultivating an acetaldehydebroadcasting $E$. coli strain in close proximity to a $\mathrm{CHO}-\mathrm{K} 1$ cell line that was engineered to have acetaldehydecontrolled expression of RipDD, a gene that encodes an 


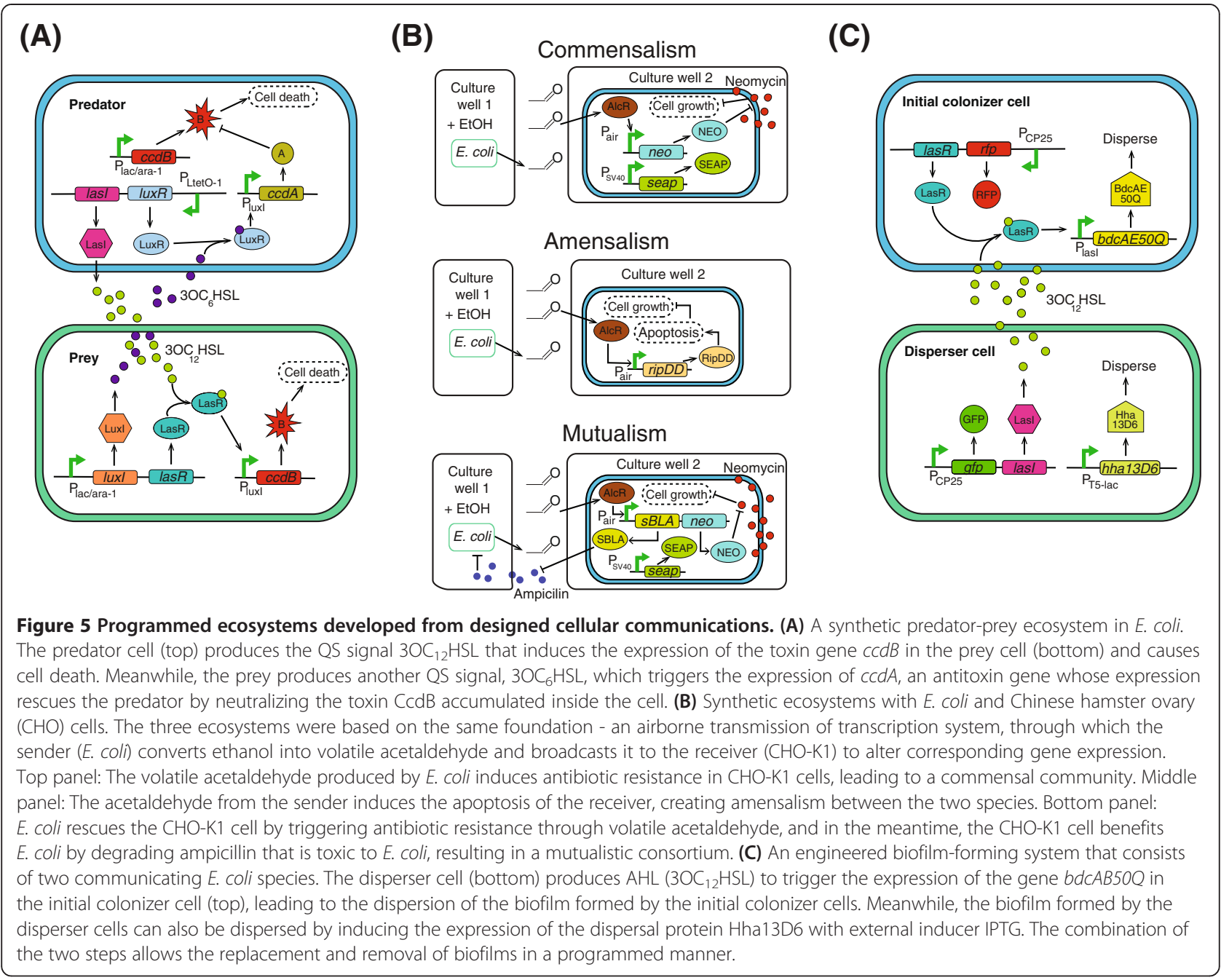

apoptosis-inducing human receptor interacting protein. As a result, the $\mathrm{CHO}-\mathrm{K} 1$ cells survive only in the absence of the $E$. coli cells because, otherwise, they induce the death of the CHO-K1 cells by producing acetaldehyde. To create a mutualistic interaction between E. coli and $\mathrm{CHO}-\mathrm{K} 1$ cells (bottom), the commensal ecosystem developed earlier (top) was modified to incorporate a mammalian beta-lactamase gene $s B L A$ under the control of the acetaldehyde-inducible promoter $\left(\mathrm{P}_{\text {air }}\right)$. Here, sBLA can be secreted to the extracellular milieu to hydrolyze the bacterial antibiotic ampicillin in the culture medium to promote the survival of co-cultured E. coli, resulting in bidirectional benefits between the two cell species. Following a similar idea, three additional types of ecosystem interactions were created, including parasitism, third party-inducible parasitism, and predator-prey interaction (not shown in Figure 5). This example demonstrated the ability of programming microbial consortia via rational design of cellular interactions by rewiring cellular communication systems, providing novel insights in understanding and programming microbial community patterns that orchestrate the complex coexistence of living systems.

In addition to programming planktonic bacterial populations, synthetic communication circuits have also been exploited in controlling complex communities such as biofilms. Hong et al. recently developed quorum-sensing circuits to program the formation and dispersal of artificial E. coli biofilms [103]. As shown in Figure 5C, the circuits have two functional parts with one belonging to the initial colonizer cell (top) and the other belonging to the disperser cell (bottom). The initial colonizer part consists of the constitutively expressed repressor gene las $R$ and its cognate promoter $\mathrm{P}_{\text {lasi }}$ that drives the expression of the biofilm dispersion gene $b d c A B 50 Q$; the disperser part is composed of the AHL-producing gene lasI that is constitutively expressed and another biofilm dispersion gene, hha13D6, controlled by external inducer IPTG. Such a design allows the disperser cell to trigger the expression of the gene $b d c A B 50 Q$ in the initial 
colonizer cell by producing AHL (3OC $12 \mathrm{HSL})$, leading to the dispersion and replacement of the biofilm formed by the initial colonizer cells. Meanwhile, the circuit in the disperser enables the biofilm formed by the dispersers to be removed with the external signal inducer IPTG. These types of functional circuits can be powerful in creating designer biofilms and enabling precise manipulation of community composition in the fields of biorefinery, medicine, and bioproduction.

\section{Conclusions}

With the advances of synthetic biology technologies and a consensus on the need for community-based functionality engineering, synthetic microbial consortia have undergone a rapid development in the past few years. This review has surveyed recent advances of engineered biological systems that utilize cell-cell communication to program bacterial group behaviors, covering both the basic communication modules and functional gene circuits that confer desired community-based dynamic behaviors.

Although there has been significant progress, the engineering of microbial communities is still in its infancy and is subject to a set of challenges. In fact, almost all synthetic circuits to date have involved many rounds of trial and error before achieving the desired functionality. Difficulties in the efficient construction of engineered circuits often stem from a lack of biological knowledge. Specifically, to facilitate gene circuit engineering, it is needed to have a deep understanding of stochasticity in gene expression [104-106], the inherent interplay between a synthetic circuit and the host organism [1], and issues related to multicellular physiology and metabolism [107]. Another big challenge arises from the technical side of synthetic biology, which includes the lack of powerful rational design platforms, limited availability of parts and modules, efficient systematic optimization strategies and toolkits, and high-throughput assays for circuit validation. Addressing the above challenges will foster our engineering capability and help to achieve the ultimate goal of efficient and reliable development of synthetic circuits with defined functionality.

Despite the challenges, the future of engineered microbial communities is bright. In fact, synthetic consortia have already started to show tremendous potential in both understanding biological questions and addressing real-world concerns. For example, extended from the programming of cellular dynamics, synthetic bacterial systems have been applied to understand ecological and evolutionary questions that are difficult to address with natural communities [108]. Towards real-world applications, bacterial consortia synthesized with designer communication modules have been used for information processing $[109,110]$, bio-computation [111], and therapeutics [112-114], as well as material and chemical productions [115-117]. There are a variety of research fields where synthetic bacterial consortia have started to play an important role: In metabolic engineering, cellular communication can be used to implement self-regulated control between cellular growth and product manufacturing in bioreactors for autonomous bioproduction. In biomedical applications, custom-tailored probiotic bacteria can be introduced into the human body to alter the composition and hence the function of the gut microbiota for disease treatment. In areas relating to the environment, biofilms and microbial consortia in soil and other natural settings can be perturbed and even reprogrammed with engineered microbes for desired purposes. We thus expect that microbial communities programmed via engineered cellular communication will become a versatile strategy in addressing both scientific and practical challenges in the near future.

\section{Abbreviations}

ADH: alcohol dehydrogenase; AHL: acyl-homoserine lactones; Al-2: autoinducer-2; AIP: auto-inducing peptide; $\mathrm{CHO}$ cells: Chinese hamster ovary cells; $\mathrm{H}_{2} \mathrm{O}_{2}$ : hydrogen peroxide; $\mathrm{Ndh}-2$ : NADH dehydrogenase II; QS: quorum sensing; SEAP: secreted alkaline phosphatase.

\section{Competing interests}

The authors declare that they have no competing interests.

\section{Authors' contributions}

$\mathrm{QH}$ and $\mathrm{TL}$ conceived the study and designed the project. WT, VC, and TL drafted the manuscript. CL analyzed the data. All authors read and approved the final manuscript.

\section{Acknowledgements}

We thank Andrew Blanchard for commenting and editing the manuscript. This work was supported by the American Heart Association (Grant No. 12SDG12090025), the Network for Computational Nanotechnology at UIUC sponsored by National Science Foundation (Grant No. 1227034), and the UIUC Research Board.

\section{Author details}

'Department of Bioengineering, University of Illinois at Urbana-Champaign, 1304 W Springfield Avenue, Urbana, IL 61801, USA. ${ }^{2}$ Institute for Genomic Biology, University of Illinois at Urbana-Champaign, 1206 W Gregory Drive, Urbana, IL 61801, USA. ${ }^{3}$ State Key Laboratory of Bioreactor Engineering, East China University of Science and Technology, 130 Meilong Road, Shanghai 200237, People's Republic of China. ${ }^{4}$ Department of Physics, University of Illinois at Urbana-Champaign, 1110 W Green Street, Urbana, IL 61801, USA.

Received: 26 August 2014 Accepted: 21 October 2014

Published online: 12 November 2014

\section{References}

1. Elowitz MB, Leibler S (2000) A synthetic oscillatory network of transcriptional regulators. Nature 403(6767):335-338

2. Gardner TS, Cantor CR, Collins JJ (2000) Construction of a genetic toggle switch in Escherichia coli. Nature 403(6767):339-342

3. Atkinson MR, Savageau MA, Myers JT, Ninfa AJ (2003) Development of genetic circuitry exhibiting toggle switch or oscillatory behavior in Escherichia coli. Cell 113(5):597-607

4. Kramer BP, Viretta AU, Daoud-El Baba M, Aubel D, Weber W, Fussenegger M (2004) An engineered epigenetic transgene switch in mammalian cells. Nat Biotechnol 22(7):867-870

5. Bayer TS, Smolke CD (2005) Programmable ligand-controlled riboregulators of eukaryotic gene expression. Nat Biotechnol 123(3):337-343 
6. Deans TL, Cantor CR, Collins JJ (2007) A tunable genetic switch based on RNAi and repressor proteins for regulating gene expression in mammalian cells. Cell 130(2):363-372

7. Friedland AE, Lu TK, Wang X, Shi D, Church G, Collins JJ (2009) Synthetic gene networks that count. Science 324(5931):1199-1202

8. Ellis T, Wang X, Collins JJ (2009) Diversity-based, model-guided construction of synthetic gene networks with predicted functions. Nat Biotechnol 27(5):465-471

9. Wu M, Su R-Q, Li X, Ellis T, Lai Y-C, Wang X (2013) Engineering of regulated stochastic cell fate determination. Proc Natl Acad Sci U S A 110(26):10610-10615

10. Fung E, Wong WW, Suen JK, Bulter T, S-g L, Liao JC (2005) A synthetic gene-metabolic oscillator. Nature 435(7038):118-122

11. Stricker J, Cookson S, Bennett MR, Mather WH, Tsimring LS, Hasty J (2008) A fast, robust and tunable synthetic gene oscillator. Nature 456(7221):516-519

12. Tigges M, Marquez-Lago TT, Stelling J, Fussenegger M (2009) A tunable synthetic mammalian oscillator. Nature 457(7227):309-312

13. Danino T, Mondragón-Palomino O, Tsimring L, Hasty J (2010) A synchronized quorum of genetic clocks. Nature 463(7279):326-330

14. Ham TS, Lee SK, Keasling JD, Arkin AP (2008) Design and construction of a double inversion recombination switch for heritable sequential genetic memory. PLoS One 3(7):e2815

15. Ajo-Franklin CM, Drubin DA, Eskin JA, Gee EP, Landgraf D, Phillips I, Silver PA (2007) Rational design of memory in eukaryotic cells. Genes Dev 21(18):2271-2276

16. Basu S, Gerchman Y, Collins CH, Arnold FH, Weiss R (2005) A synthetic multicellular system for programmed pattern formation. Nature 434(7037):1130-1134

17. Kobayashi H, Kærn M, Araki M, Chung K, Gardner TS, Cantor CR, Collins JJ (2004) Programmable cells: interfacing natural and engineered gene networks. Proc Natl Acad Sci U S A 101(22):8414-8419

18. You L, Cox RS, Weiss R, Arnold FH (2004) Programmed population control by cell-cell communication and regulated killing. Nature 428(6985):868-871

19. Guet CC, Elowitz MB, Hsing W, Leibler S (2002) Combinatorial synthesis of genetic networks. Science 296(5572):1466-1470

20. Rackham O, Chin JW (2005) Cellular logic with orthogonal ribosomes. J Am Chem Soc 127(50):17584-17585

21. Anderson JC, Voigt CA, Arkin AP (2007) Environmental signal integration by a modular AND gate. Mol Syst Biol 3:133

22. Win MN, Smolke CD (2008) Higher-order cellular information processing with synthetic RNA devices. Science 322(5900):456-460

23. Basu S, Mehreja R, Thiberge S, Chen M-T, Weiss R (2004) Spatiotemporal control of gene expression with pulse-generating networks. Proc Natl Acad Sci U S A 101(17):6355-6360

24. Hooshangi S, Thiberge S, Weiss R (2005) Ultrasensitivity and noise propagation in a synthetic transcriptional cascade. Proc Natl Acad Sci U S A 102(10):3581-3586

25. Sohka T, Heins RA, Phelan RM, Greisler JM, Townsend CA, Ostermeier M (2009) An externally tunable bacterial band-pass filter. Proc Natl Acad Sci U S A 106(25):10135-10140

26. Church GM, Elowitz MB, Smolke CD, Voigt CA, Weiss R (2014) Realizing the potential of synthetic biology. Nat Rev Mol Cell Biol 15(4):289-94

27. Khalil AS, Collins JJ (2010) Synthetic biology: applications come of age. Nat Rev Genet 11(5):367-379

28. Ruder WC, Lu T, Collins JJ (2011) Synthetic biology moving into the clinic. Science 333(6047):1248-1252

29. Weber W, Fussenegger M (2011) Emerging biomedical applications of synthetic biology. Nat Rev Genet 13(1):21-35

30. Gibson DG, Young L, Chuang R-Y, Venter JC, Hutchison CA, Smith HO (2009) Enzymatic assembly of DNA molecules up to several hundred kilobases. Nat Methods 6(5):343-345

31. Engler C, Kandzia R, Marillonnet S (2008) A one pot, one step, precision cloning method with high throughput capability. PLoS One 3(11):e3647

32. Zhang Y, Werling U, Edelmann W (2012) SLiCE: a novel bacterial cell extract-based DNA cloning method. Nucleic Acids Res 40(8):e55-e55

33. Quan J, Tian J (2011) Circular polymerase extension cloning for high-throughput cloning of complex and combinatorial DNA libraries. Nat Protoc 6(2):242-251

34. Wang HH, Isaacs FJ, Carr PA, Sun ZZ, Xu G, Forest CR, Church GM (2009) Programming cells by multiplex genome engineering and accelerated evolution. Nature 460(7257):894-898

35. Ellis HM, Yu D, DiTizio T (2001) High efficiency mutagenesis, repair, and engineering of chromosomal DNA using single-stranded oligonucleotides. Proc Natl Acad Sci U S A 98(12):6742-6746
36. Jiang W, Bikard D, Cox D, Zhang F, Marraffini LA (2013) RNA-guided editing of bacterial genomes using CRISPR-Cas systems. Nat Biotechnol 31(3):233-239

37. Gaj T, Gersbach CA, Barbas CF III (2013) ZFN, TALEN, and CRISPR/Cas-based methods for genome engineering. Trends Biotechnol 31(7):397-405

38. Slusarczyk AL, Lin A, Weiss R (2012) Foundations for the design and implementation of synthetic genetic circuits. Nat Rev Genet 13(6):406-420

39. Salis HM, Mirsky EA, Voigt CA (2009) Automated design of synthetic ribosome binding sites to control protein expression. Nat Biotechnol 27(10):946-950

40. Hillson NJ, Rosengarten RD, Keasling JD (2011) j5 DNA assembly design automation software. ACS Synth Biol 1(1):14-21

41. Lou C, Stanton B, Chen Y-J, Munsky B, Voigt CA (2012) Ribozyme-based insulator parts buffer synthetic circuits from genetic context. Nat Biotechnol 30(11):1137-1142

42. Smolke CD (2009) Building outside of the box: iGEM and the BioBricks Foundation. Nat Biotechnol 27(12):1099-1102

43. Chuang JS (2012) Engineering multicellular traits in synthetic microbial populations. Curr Opin Chem Biol 16(3):370-378

44. Brenner K, You L, Arnold FH (2008) Engineering microbial consortia: a new frontier in synthetic biology. Trends Biotechnol 26(9):483-489

45. Pai A, Tanouchi $Y$, Collins CH, You L (2009) Engineering multicellular systems by cell-cell communication. Curr Opin Biotechnol 20(4):461-470

46. Payne S, You L (2013) Engineered cell-cell communication and its applications. Adv Biochem Eng Biotechnol 146:97-121

47. Tsao C-Y, Quan DN, Bentley WE (2012) Development of the quorum sensing biotechnological toolbox. Curr Opin Chem Eng 1(4):396-402

48. Teuscher C, Grecu C, Lu T, Weiss R (2011) Challenges and promises of nano and bio communication networks. In: Networks on Chip (NoCS), 2011 Fifth IEEE/ACM International Symposium on. IEEE, Pittsburgh, pp 247-254

49. Liu C, Fu X, Liu L, Ren X, Chau CK, Li S, Xiang L, Zeng H, Chen G, Tang L-H (2011) Sequential establishment of stripe patterns in an expanding cell population. Science 334(6053):238-241

50. Brenner K, Karig DK, Weiss R, Arnold FH (2007) Engineered bidirectional communication mediates a consensus in a microbial biofilm consortium. Proc Natl Acad Sci U S A 104(44):17300-17304

51. Weber W, Daoud-El Baba M, Fussenegger M (2007) Synthetic ecosystems based on airborne inter- and intra-kingdom communication. Proc Natl Acad Sci U S A 104(25):10435-10440

52. Balagaddé FK, Song H, Ozaki J, Collins CH, Barnet M, Arnold FH, Quake SR, You L (2008) A synthetic Escherichia coli predator-prey ecosystem. Mol Syst Biol 4:187

53. O'Toole G, Kaplan HB, Kolter R (2000) Biofilm formation as microbial development. Annu Rev Microbiol 54(1):49-79

54. Stoodley P, Sauer K, Davies D, Costerton JW (2002) Biofilms as complex differentiated communities. Annu Rev Microbiol 56(1):187-209

55. Consortium HMP (2012) Structure, function and diversity of the healthy human microbiome. Nature 486(7402):207-214

56. Cho I, Blaser MJ (2012) The human microbiome: at the interface of health and disease. Nat Rev Genet 13(4):260-270

57. Costerton J, Stewart PS, Greenberg E (1999) Bacterial biofilms: a common cause of persistent infections. Science 284(5418):1318-1322

58. Stewart PS, William Costerton J (2001) Antibiotic resistance of bacteria in biofilms. Lancet 358(9276):135-138

59. Koizumi S, Endo T, Tabata K, Ozaki A (1998) Large-scale production of UDP-galactose and globotriose by coupling metabolically engineered bacteria. Nat Biotechnol 16(9):847-850

60. Minty JJ, Singer ME, Scholz SA, Bae C-H, Ahn J-H, Foster CE, Liao JC, Lin XN (2013) Design and characterization of synthetic fungal-bacterial consortia for direct production of isobutanol from cellulosic biomass. Proc Natl Acad Sci U S A 110(36):14592-14597

61. Chen M-T, Weiss R (2005) Artificial cell-cell communication in yeast Saccharomyces cerevisiae using signaling elements from Arabidopsis thaliana. Nat Biotechnol 23(12):1551-1555

62. Williams TC, Nielsen LK, Vickers CE (2013) Engineered quorum sensing using pheromone-mediated cell-to-cell communication in Saccharomyces cerevisiae. ACS Synth Biol 2(3):136-149

63. Bassler BL, Losick R (2006) Bacterially speaking. Cell 125(2):237-246

64. Waters CM, Bassler BL (2005) Quorum sensing: cell-to-cell communication in bacteria. Annu Rev Cell Dev Biol 21:319-346

65. Engebrecht J, Nealson K, Silverman M (1983) Bacterial bioluminescence: isolation and genetic analysis of functions from Vibrio fischeri. Cell 32(3):773-781 
66. Davies DG, Parsek MR, Pearson JP, Iglewski BH, Costerton J, Greenberg E (1998) The involvement of cell-to-cell signals in the development of a bacterial biofilm. Science 280(5361):295-298

67. De Kievit TR, Iglewski BH (2000) Bacterial quorum sensing in pathogenic relationships. Infect Immun 68(9):4839-4849

68. Latifi A, Winson MK, Foglino M, Bycroft BW, Stewart GS, Lazdunski A Williams P (1995) Multiple homologues of LuxR and Luxl control expression of virulence determinants and secondary metabolites through quorum sensing in Pseudomonas aeruginosa PAO1. Mol Microbiol 17(2):333-343

69. Thomson N, Crow M, McGowan S, Cox A, Salmond G (2000) Biosynthesis of carbapenem antibiotic and prodigiosin pigment in Serratia is under quorum sensing control. Mol Microbiol 36(3):539-556

70. Kleerebezem M (2004) Quorum sensing control of lantibiotic production; nisin and subtilin autoregulate their own biosynthesis. Peptides 25(9):1405-1414

71. Fuqua C, Parsek MR, Greenberg EP (2001) Regulation of gene expression by cell-to-cell communication: acyl-homoserine lactone quorum sensing. Annu Rev Genet 35(1):439-468

72. Thiel V, Kunze B, Verma P, Wagner-Döbler I, Schulz S (2009) New structural variants of homoserine lactones in bacteria. Chem Bio Chem 10(11):1861-1868

73. Fuqua WC, Winans SC, Greenberg EP (1994) Quorum sensing in bacteria: the LuxR-Luxl family of cell density-responsive transcriptional regulators. J Bacteriol 176(2):269

74. Nealson K, Hastings JW (1979) Bacterial bioluminescence: its control and ecological significance. Microbiol Rev 43(4):496

75. Pesci EC, Pearson JP, Seed PC, Iglewski BH (1997) Regulation of las and rhl quorum sensing in Pseudomonas aeruginosa. J Bacteriol 179(10):3127-3132

76. Welch M, Todd DE, Whitehead NA, McGowan SJ, Bycroft BW, Salmond GP (2000) N-acyl homoserine lactone binding to the CarR receptor determines quorum-sensing specificity in Erwinia. EMBO J 19(4):631-641

77. Minogue TD, Trebra MW, Bernhard F, Bodman SB (2002) The autoregulatory role of EsaR, a quorum-sensing regulator in Pantoea stewartii ssp. stewartii: evidence for a repressor function. Mol Microbiol 44(6):1625-1635

78. Novick RP, Geisinger E (2008) Quorum sensing in Staphylococci. Annu Rev Genet 42:541-564

79. Hancock LE, Perego M (2004) The Enterococcus faecalis fsr two-component system controls biofilm development through production of gelatinase. J Bacteriol 186(17):5629-5639

80. Pestova E, Håvarstein L, Morrison D (1996) Regulation of competence for genetic transformation in Streptococcus pneumoniae by an auto-induced peptide pheromone and a two-component regulatory system. Mol Microbiol 21(4):853-862

81. Winzer K, HARDIE K, Williams P (2003) LuXS and autoinducer-2: their contribution to quorum. Adv Appl Microbiol 53:291

82. Pereira CS, Thompson JA, Xavier KB (2013) Al-2-mediated signalling in bacteria. FEMS Microbiol Rev 37(2):156-181

83. Prindle A, Samayoa P, Razinkov I, Danino T, Tsimring LS, Hasty J (2012) A sensing array of radically coupled genetic 'biopixels'. Nature 481(7379):39-44

84. Pesci EC, Milbank JB, Pearson JP, McKnight S, Kende AS, Greenberg EP, Iglewski BH (1999) Quinolone signaling in the cell-to-cell communication system of Pseudomonas aeruginosa. Proc Natl Acad Sci U S A 96(20):11229-11234

85. Ryan RP, Dow JM (2011) Communication with a growing family: diffusible signal factor (DSF) signaling in bacteria. Trends Microbiol 19(3):145-152

86. Holden MT, Ram Chhabra S, De Nys R, Stead P, Bainton NJ, Hill PJ, Manefield M, Kumar N, Labatte M, England D (1999) Quorum-sensing cross talk: isolation and chemical characterization of cyclic dipeptides from Pseudomonas aeruginosa and other gram-negative bacteria. Mol Microbiol 33(6):1254-1266

87. Tommonaro G, Abbamondi GR, lodice C, Tait K, De Rosa S (2012) Diketopiperazines produced by the halophilic archaeon, Haloterrigena hispanica, activate AHL bioreporters. Microb Ecol 63(3):490-495

88. Flavier AB, Clough SJ, Schell MA, Denny TP (1997) Identification of 3-hydroxypalmitic acid methyl ester as a novel autoregulator controlling virulence in Ralstonia solanacearum. Mol Microbiol 26(2):251-259

89. Chen H, Fujita M, Feng Q, Clardy J, Fink GR (2004) Tyrosol is a quorum-sensing molecule in Candida albicans. Proc Natl Acad Sci U S A 101(14):5048-5052

90. Lee JH, Lee J (2010) Indole as an intercellular signal in microbial communities. FEMS Microbiol Rev 34(4):426-444

91. Smith R, Tan C, Srimani JK, Pai A, Riccione KA, Song H, You L (2014) Programmed Allee effect in bacteria causes a tradeoff between population spread and survival. Proc Natl Acad Sci U S A 111(5):1969-1974
92. Stephens PA, Sutherland WJ, Freckleton RP (1999) What is the Allee effect? Oikos 87:185-190

93. Shapiro JA (1998) Thinking about bacterial populations as multicellular organisms. Annu Rev Microbiol 52(1):81-104

94. Parsek MR, Greenberg E (2005) Sociomicrobiology: the connections between quorum sensing and biofilms. Trends Microbiol 13(1):27-33

95. Gurdon J, Bourillot P-Y (2001) Morphogen gradient interpretation. Nature 413(6858):797-803

96. Wolpert $L$ (1969) Positional information and the spatial pattern of cellular differentiation. J Theor Biol 25(1):1-47

97. Payne S, Li B, Cao Y, Schaeffer D, Ryser MD, You L (2014) Temporal control of self-organized pattern formation without morphogen gradients in bacteria. Mol Syst Biol 9:697

98. Turing AM (1952) The chemical basis of morphogenesis. Phil Trans R Soc Lond B 237(641):37-72

99. Tabor JJ, Salis HM, Simpson ZB, Chevalier AA, Levskaya A, Marcotte EM, Voigt CA, Ellington AD (2009) A synthetic genetic edge detection program. Cell 137(7):1272-1281

100. Gambetta GA, Lagarias JC (2001) Genetic engineering of phytochrome biosynthesis in bacteria. Proc Natl Acad Sci U S A 98(19):10566-10571

101. Levskaya A, Chevalier AA, Tabor JJ, Simpson ZB, Lavery LA, Levy M, Davidson EA, Scouras A, Ellington AD, Marcotte EM (2005) Synthetic biology: engineering Escherichia coli to see light. Nature 438(7067):441-442

102. Faust K, Raes J (2012) Microbial interactions: from networks to models. Nat Rev Microbiol 10(8):538-550

103. Hong SH, Hegde M, Kim J, Wang X, Jayaraman A, Wood TK (2012) Synthetic quorum-sensing circuit to control consortial biofilm formation and dispersal in a microfluidic device. Nat Commun 3:613

104. Lu T, Hasty J, Wolynes PG (2006) Effective temperature in stochastic kinetics and gene networks. Biophys J 91(1):84-94

105. Lu T, Shen T, Zong C, Hasty J, Wolynes PG (2006) Statistics of cellular signal transduction as a race to the nucleus by multiple random walkers in compartment/ phosphorylation space. Proc Natl Acad Sci U S A 103(45):16752-16757

106. Lu T, Shen T, Bennett MR, Wolynes PG, Hasty J (2007) Phenotypic variability of growing cellular populations. Proc Natl Acad Sci U S A 104(48):18982-18987

107. Mao J, Blanchard AE, Lu T (2014) Slow and steady wins the race: a bacterial exploitative competition strategy in fluctuating environments. ACS Synth Biol doi:10.1021/sb4002008

108. Song H, Payne S, Gray M, You L (2009) Spatiotemporal modulation of biodiversity in a synthetic chemical-mediated ecosystem. Nat Chem Biol 5(12):929-935

109. Prindle A, Selimkhanov J, Li H, Razinkov I, Tsimring LS, Hasty J (2014) Rapid and tunable post-translational coupling of genetic circuits. Nature 508(7496):387-91

110. Qi H, Blanchard A, Lu T (2013) Engineered genetic information processing circuits. Wiley Interdiscip Rev Syst Biol Med 5(3):273-287

111. Tamsir A, Tabor JJ, Voigt CA (2011) Robust multicellular computing using genetically encoded NOR gates and chemical 'wires'. Nature 469(7329):212-215

112. Saeidi N, Wong CK, Lo TM, Nguyen HX, Ling H, Leong SSJ, Poh CL, Chang MW (2011) Engineering microbes to sense and eradicate Pseudomonas aeruginosa, a human pathogen. Mol Syst Biol 7:521

113. Hwang IY, Tan MH, Koh E, Ho CL, Poh CL, Chang MW (2013) Reprogramming microbes to be pathogen-seeking killers. ACS Synth Biol 3(4):228-237

114. Duan F, March JC (2010) Engineered bacterial communication prevents Vibrio cholerae virulence in an infant mouse model. Proc Natl Acad Sci U S A 107(25):11260-11264

115. Chen AY, Deng Z, Billings AN, Seker UO, Lu MY, Citorik RJ, Zakeri B, Lu TK (2014) Synthesis and patterning of tunable multiscale materials with engineered cells. Nat Mater 13:515-523

116. Nocadello S, Swennen EF (2012) The new pLAI (lux regulon based auto-inducible) expression system for recombinant protein production in Escherichia coli. Microb Cell Fact 11(1):1-10

117. Tsao C-Y, Hooshangi S, Wu H-C, Valdes JJ, Bentley WE (2010) Autonomous induction of recombinant proteins by minimally rewiring native quorum sensing regulon of E. coli. Metab Eng 12(3):291-297

doi:10.1186/s40643-014-0024-6

Cite this article as: Kong et al:: Programming the group behaviors of bacterial communities with synthetic cellular communication.

Bioresources and Bioprocessing 2014 1:24. 